Proc. Indian Acad. Sci. (Chem. Sci.), Vol. 105, No. 1, February 1993, pp. 53-62.

(C) Printed in India.

\title{
Is tetramethyleneethane a ground state triplet?
}

\author{
APARNA CHAKRABARTI," I D L ALBERT, ${ }^{\text {S }}$ RAMASESHA*a, \\ $S^{S}$ LALITHA $^{b}$ and JAYARAMAN CHANDRASEKHAR ${ }^{* b}$ \\ aSolid State and Structural Chemistry Unit, and \\ ${ }^{\text {b}}$ Department of Organic Chemistry, Indian Institute of Science, Bangalore 560012, India
}

MS received 7 May 1992; revised 24 September 1992

\begin{abstract}
We have examined a number of possible ways by which tetramethyleneethane (TME) can be a ground state triplet, as claimed by experimental studies, in violation of Ovchinnikov's theorem for alternant hydrocarbons of equal bond lengths. Model exact $\pi$ calculations of the low-lying states of TME, 3,4-dimethylenefuran and 3,4-dimethylenepyrrole were carried out using a diagrammatic valence bond approach. The calculations failed to yield a triplet ground state even after (a) tuning of electron correlation, (b) breaking alternancy symmetry, and (c) allowing for geometric distortions. In contrast to earlier studies of fine structure constants in other conjugated systems, the computed $D$ and $E$ values of all the low-lying triplet states of TME for various geometries are at least an order of magnitude different from the experimentally reported values. Incorporation of $\sigma-\pi$ mixing by means of UHF MNDO calculations is found to favour a singlet ground state even further. A reinterpretation of the experimental results of TME is therefore suggested to resolve the conflict.
\end{abstract}

Keywords. Tetramethyleneethane; biradical; singlet-triplet gap; MO calculations; electronic structure.

\section{Introduction}

The ground state of tetramethyleneethane (TME) has been a subject of considerable controversy in recent years (Borden and Davidson 1977; Borden 1982; Lahti et al 1985; Dowd et al 1986, 1987; Du and Borden 1987; Choi et al 1988). The Huckel model predicts that the lowest singlet and the triplet states are degenerate. However, this degeneracy is lifted on introducing electron correlations, and within the alternant description with uniform bond lengths the ground state is a singlet (Borden 1982). In fact, within any $\pi$ electron model assuming uniform bond lengths, Ovchinnikov's exact theorem leads to a singlet ground state for all alternant hydrocarbons with equal numbers of starred and unstarred carbon atoms such as TME (Ovchinnikov 1971). All-valence and all-electron calculations also have led to the same conclusion. Various semiempirical (INDO/S) (Lahti et al 1985), (AM1) (Radhakrishnan 1991) and $a b$ initio (Borden and Davidson 1977; Du and Borden 1987) calculations predict a singlet ground state for TME, as long as some electron correlation is included. In striking contrast to these qualitative and quantitative theoretical results, recent

* For correspondence 
experimental studies on TME as well as on related model diradicals seem to indicate that the ground state is indeed a triplet (Dowd et al 1986, 1987; Roth et al 1987).

There may be many reasons for the apparent failure of theory. Electron correlation needs to be adequately taken into account for any meaningful computation of the energies of closely spaced levels of a conjugated system (Borden 1982). A comprehensive examination of the role of electron correlation in TME is therefore warranted. Also, TME does not strictly possess alternancy symmetry because of differing chemical environments of the central and terminal carbon atoms. Geometric distortions, such as unequal bond lengths as well as twisting about the central $\mathrm{C}-\mathrm{C}$ bond may also be responsible for the observed triplet ground state. Finally, the breakdown of Ovchinnikov's theorem for TME may result from significant $\sigma-\pi$ interactions. While some of these factors have been considered before, especially the role of twisting about the central bond (Dowd et al 1987; Du and Borden 1987; Choi et al 1988), we have now carried out a detailed examination of all the possible sources of conflict between experiment and theory mentioned above. In the present study, employing a diagrammatic valence bond (VB) method for solving exactly the model Hamiltonians, we have examined the effect of breaking the alternancy symmetry, tuning electron correlations and varying the geometries on the spin multiplicity of the ground state in TME. The effect of $\sigma-\pi$ polarisation has also been studied by means of the UHF MNDO method (Dewar and Thiel 1977).

\section{Computational details}

The nontrivial $\pi$-electron models we consider are the Pariser-Parr-Pople (PPP) model and the Hubbard model. The PPP model is given by (Pariser and Parr 1953; Pople 1953):

$$
\begin{aligned}
H_{\mathrm{PPP}}= & \sum_{i \sigma} \varepsilon_{i} a_{i \sigma}^{*} a_{i \sigma}+\sum_{\langle i j\rangle, \sigma} t_{\langle i j\rangle}\left(a_{i \sigma}^{*} a_{j \sigma}+h . c .\right)+ \\
& \sum_{i} U_{i} \hat{n}_{i, \sigma} \hat{n}_{i,-\sigma}+\sum_{i<j} V_{i j}\left(\hat{n}_{i}-z_{i}\right)\left(\hat{n}_{j}-z_{j}\right),
\end{aligned}
$$

where $\varepsilon_{i}$ is the orbital energy of $p_{z}$ orbital on the $i$ th carbon atom and $t_{\langle i j\rangle}$ is the Huckel resonance integral between the $i$ th and the $j$ th carbon atoms, $a_{i, \sigma}^{*}\left(a_{i, \sigma}\right)$ creates (annihilates) an electron in the $p_{z}$ orbital on site $i$ with spin $\sigma$. The parameter $U$ is the on-site repulsion energy, $V_{i j}$ is the intersite interaction energy and $z_{i}$ is the occupancy of the $i$ th $p_{z}$ orbital that leaves the atom on site $i$ neutral. The intersite repulsion $V_{i j}$ is parametrized as (Ohno 1964):

$$
V_{i j}=14.397\left\{\left[28 \cdot 794 /\left(U_{i}+U_{j}\right)\right]^{2}+r_{i j}^{2}\right\}^{-1 / 2} \text {. }
$$

The PPP model has been extensively applied to the $\pi$-electron systems within a valence bond (VB) formalism that allows a model exact solution in systems with upto $12 \pi$-orbitals, without any additional symmetry adaptation (Albert et al 1991; Ramasesha et al 1991). These earlier studies have yielded a very reliable set of molecular parameters which predict, besides accurate excitation spectra, properties such as transition dipoles, spin densities (Soos and Ramasesha 1983; Ramasesha and 


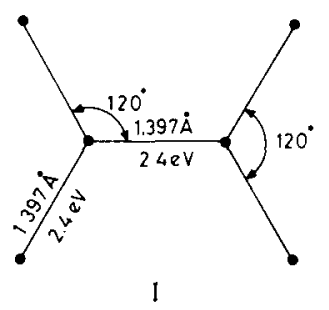

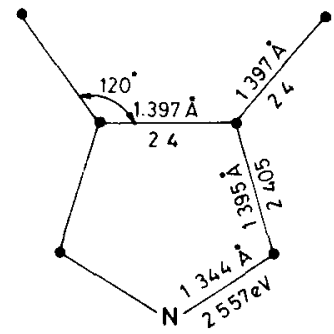

II

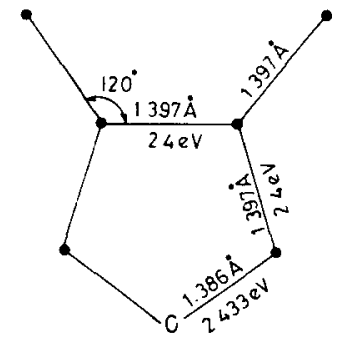

III

Figure 1. Structures, idealized bond distances, bond angles and resonance integrals in tetramethyleneethane (I), 3,4-dimethylenepyrrole (II) and 3,4-dimethylenefuran (III).

Soos 1984a), aromatic ring currents (Kuwajima and Soos 1987) and fine structure constants in the high spin states (Ramasesha and Soos 1984b) that are in good agreement with experiment. Therefore, we first computed within the PPP model the energies of several low-lying states in different spin spaces of TME (figure 1, I). A constant $\mathrm{C}-\mathrm{C}$ bond length of $1.397 \AA$ (the value in benzene) was chosen in these initial calculations.

Next, we examined the importance of electron correlation in determining the ground state of TME. The Hubbard model comprising the first three terms in (1) is the most appropriate for this purpose. The model is heuristic since the screening of electron interactions in these systems is not anywhere nearly as efficient as in metals. However, the Hubbard parameter $U / t$ which is the ratio of the on-site correlation strength to the Huckel resonance integral, can be continuously varied and provides insights into the behaviour of the system at different correlation energies (Hubbard 1963).

We then varied the site energies of the PPP model for the central carbon atoms, to follow the effect of breakdown of alternancy symmetry. The singlet-triplet gap was computed for a range of reasonable values of the site energies.

Ovchinnikov's theorem is not valid if the central and terminal $\mathrm{C}-\mathrm{C}$ bond lengths become unequal. Geometry optimization was carried out to see whether the triplet is selectively stabilized in the equilibrium geometry. To optimize the bond distances, we first calculate the bond orders of each of the bonds in the two states and use Coulson's (1961) bond order-bond distance relation:

$$
x=s-\{[s-d] /[1+K(1-P)] / P\},
$$

where $x$ is the new bond distance, $s$ and $d$ are the single and double bond distances ( $1.3403 \AA$ and $1.4537 \AA$ respectively), and $K$ is the ratio of the force constant of the 
single bond to that of the double bond which is taken to be 0.506 . The revised resonance integrals for these bond distances are computed following. Ducasse et al (1982):

$$
t_{i j}=-2 \cdot 40+3 \cdot 20\left(r_{i j}-1 \cdot 397\right)
$$

where $t_{i j}$ is in $\mathrm{eV}$ and $r_{i j}$ is the distance in Angstroms between the $i$ th and the $j$ th carbon atoms. We iterate on the bond distances and bond orders until self-consistency is achieved.

Besides changes in bond lengths, the effect of twisting the molecule about the central bond was also considered. Further, in the lowest triplet states the fine structure constants for spin-spin interactions, $D$ and $E$, have been calculated for all twist angles.

We have also studied cyclic conjugated systems related to TME-containing hetero atoms, namely, 3,4-dimethylenepyrrole (figure 1, II) and 3,4-dimethylenefuran (III) within the PPP model for comparison with earlier studies (Du et al 1986; Stone et al 1986; Zilm et al 1987).

While exact solutions are possible within the $\pi$-electron models, they are open to the criticism that $\sigma-\pi$ separability may not be completely justified. To account for the contributions due to the $\sigma$ framework, we have carried out UHF-MNDO calculations with full geometry optimization on both singlet and triplet TME and its analogues. This simple computational procedure predicts the correct ground state in numerous diradicaloid systems (Lalitha 1992).

\section{Results and discussion}

In tetramethyleneethane, if the marginal differences in the chemical environments of the central and terminal carbon atoms are neglected, the system will be an alternant system in the neutral state and from Ovchinnikov's (1971) theorem, the system will be in the singlet ground state. Since Ovchinnikov's theorem follows from that of Lieb and Mattis (1962), it is valid when all the resonance integrals are also identical. The exact solution of the PPP model with molecular parameters indeed shows this to be the case (figure 1, table 1). We also note that the first excited singlet state is higher in energy than the two lowest energy triplet states.

Calculations on the Hubbard model also show that the ground state is a singlet for all nonzero values of $U / t$ (figure 2). When the correlation energy is zero $(U / t=0)$,

Table 1. Exact PPP energies (without geometry optimization) of tetramethyleneethane, 3,4-dimethylenepyrrole and 3,4-dimethylenefuran.

\begin{tabular}{lccc}
\hline Diradical & State & $\begin{array}{c}\text { Singlet energy } \\
(\mathrm{eV})\end{array}$ & $\begin{array}{c}\text { Triplet energy } \\
(\mathrm{eV})\end{array}$ \\
\hline Tetramethyleneethane & 1 & 0.0 & 0.0908 \\
& 2 & 2.9483 & 2.2516 \\
3,4-Dimethylenepyrrole & 1 & 0.0 & 0.6936 \\
& 2 & 2.5791 & 1.9320 \\
3,4-Dimethylenefuran & 1 & 0.0 & 0.2639 \\
& 2 & 2.5450 & 1.9595 \\
\hline
\end{tabular}




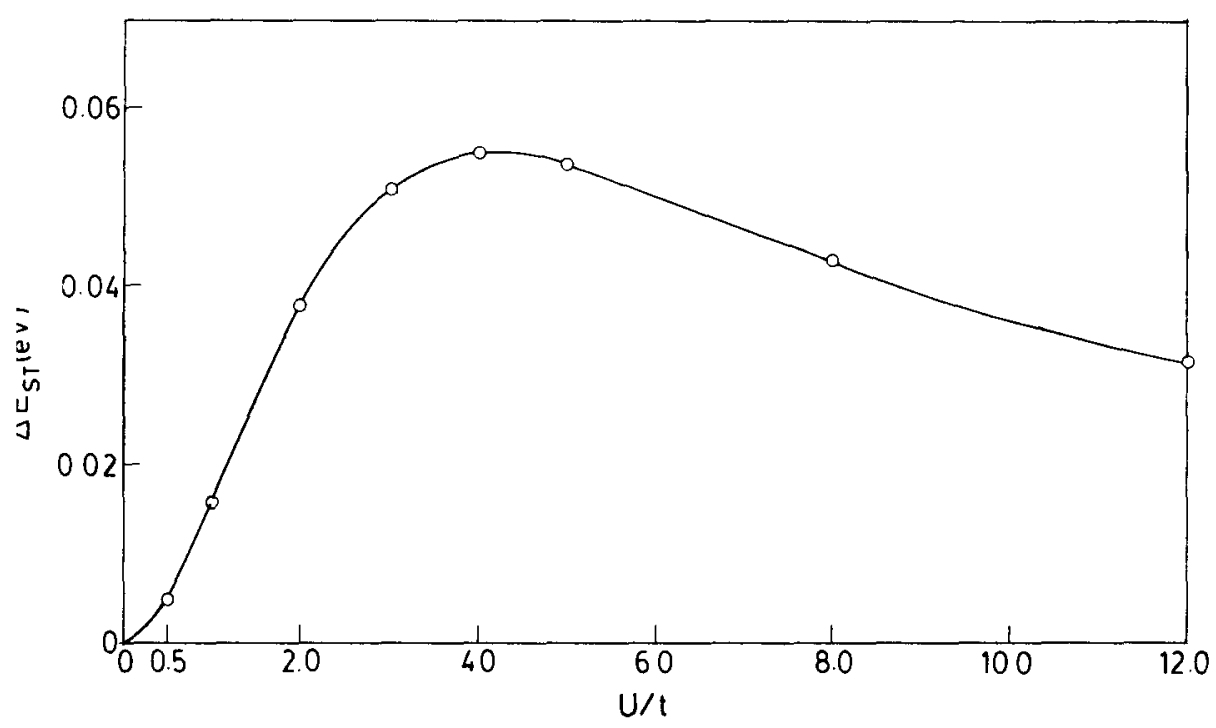

Figure 2. Variation in the singlet-triplet gap as a function of the Hubbard correlation strength $U$ in units of $t$, in tetramethyleneethane.

corresponding to the Huckel model, the lowest triplet is degenerate with the lowest singlet state. Introduction of correlations lifts this degeneracy since in the singlet state the electrons can delocalize with facility while in the triplet state, not all the electron hops are permitted because of Pauli exclusion principle. The singlet-triplet gap is largest when the correlation energy is comparable to the resonance integral and in our case peaks at $U / t=4 \cdot 0$. However, as the electron correlation strength is increased, the delocalization energy comes down and in the limit of infinite correlation strength, the atomic limit is reached, where the singlet and the triplet and all other possible spin states again become degenerate.

The alternancy symmetry is broken in the PPP model by introducing different site energies for the two types of carbon centres. In tetramethyleneethane, the central carbon atoms are expected to have a slightly higher orbital energy than the terminal carbon atoms due to the greater stability associated with tertiary carbenium ions relative to primary carbenium ions. We have studied the excitation spectrum of the PPP Hamiltonian after introducing a positive site energy at the central sites, relative to the terminal sites. We find that the singlet-triplet gap is hardly affected even for as large a site energy difference as $2.0 \mathrm{eV}$ (table 2). The singlet state continues to be the ground state for all reasonable values of the site energies. This is also in conformity with the exact PPP studies on conjugated polymers predicted to be in the high spin ground state from Ovchinnikov's theorem (Sinha et al 1990).

Within the $\pi$-electron model, Ovchinnikov's theorem may be violated if the resonance integrals are nonequivalent. This may arise from inequivalent bond distances in the system. We therefore optimized the bond lengths in TME and computed the singlet-triplet energy difference (table 3 ). The bond distance initially assumed is indeed close to the optimised value. The middle bond, after optimization is slightly elongated while the terminal bonds are shortened. Even in the optimized geometries, the triplet state lies above the singlet state by $0.085 \mathrm{eV}$. 
Table 2. Exact PPP energies of tetramethyleneethane with different site energies $\varepsilon$ on the central carbon atoms.

\begin{tabular}{lcc}
$\begin{array}{l}\varepsilon \\
(\mathrm{eV})\end{array}$ & $\begin{array}{c}\text { Singlet energies } \\
(\mathrm{eV})\end{array}$ & $\begin{array}{c}\text { Triplet energies } \\
(\mathrm{eV})\end{array}$ \\
\hline $0.0^{*}$ & 0.0 & 0.0852 \\
& 2.9820 & 2.2739 \\
0.5 & 0.0 & 0.0876 \\
& 2.9816 & 2.2727 \\
2.0 & 0.0 & 0.0801 \\
& 2.8943 & 2.2758 \\
\hline
\end{tabular}

*Geometry optimized energies.

Table 3. Self consistent calculation of the bond order $\left(P_{i j}\right)$ and bond distances $\left(R_{i j}\right)$ of tetramethyleneethane in the singlet and triplet states.

\begin{tabular}{lcccccc}
\hline \multirow{2}{*}{$\begin{array}{l}\text { No. of } \\
\text { iterations }\end{array}$} & $\begin{array}{c}\text { Bond } \\
i-j\end{array}$ & \multicolumn{2}{c}{ Singlet state } & & \multicolumn{2}{c}{ Triplet state } \\
\cline { 3 - 6 } \cline { 5 - 6 } & $R_{i j}$ & $P_{i j}$ & & $R_{i j}$ & $P_{i j}$ \\
\hline 0 & $1-2$ & 1.3970 & 1.247 & & 1.3970 & 1.262 \\
& $2-5$ & 1.3970 & 0.634 & & 1.3970 & 0.568 \\
& $1-2$ & 1.3947 & 1.250 & & 1.3943 & 1.265 \\
& $2-5$ & 1.4045 & 0.625 & & 1.4064 & 0.558 \\
& $1-2$ & 1.3944 & 1.250 & & 1.3943 & 1.265 \\
& $2-5$ & 1.4047 & 0.625 & & 1.4067 & 0.558 \\
\hline
\end{tabular}

We have also studied within the PPP model the effect of twisting the molecule about the central bond on the excitation spectrum. Experimentally, both the conformationally free TME (Dowd et al 1986) and the rigid 2,3-dimethylenecyclohexa1,3-diene diradical (Dowd et al 1987) have been suggested to be ground state triplets. $A b$ initio calculations (Du and Borden 1987) remain discordant with the experimental conclusion by predicting a singlet ground state irrespective of the twist angle about the central bond. Our results confirm the earlier theoretical finding. Figure 3 shows a plot of the energies of the two lowest energy singlet and triplet states as a function of the twist angle. The singlet state continues to be the ground state for all twist angles. However, the lowest singlet and triplet states become degenerate when the twist angle is $90^{\circ}$. It is interesting to note that the first excited singlet and the first excited triplet states also become degenerate for this twist angle. This happens because for the $90^{\circ}$ twist, the two parts of the molecule are disconnected leading to two possibilities. The lower energy possibility corresponds to the two parts containing 3 electrons each with the spin of each fragment being half. These two fragments can lead to spin 0 and spin 1 states of equal energy. The higher energy triplet and singlet states are derived from the ionic states and correspond to the case in which the two fragments have unequal numbers of electrons (two and four). The spin of each fragment in this case should be one if the states with total spin 0 and 1 should be degenerate. 


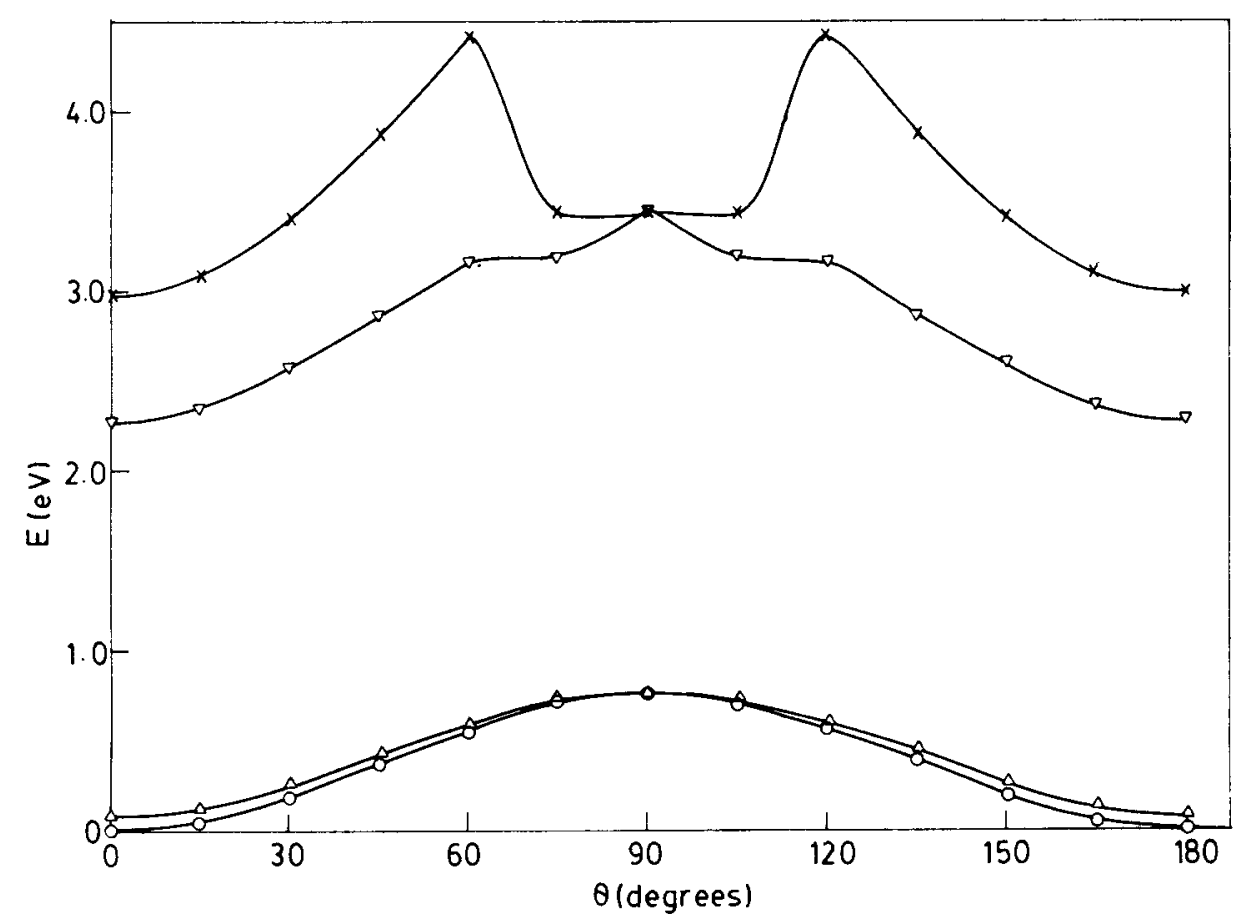

Figure 3. Dependence of the exact PPP state energies on the twist angle $\theta$ for rotation about the central bond in tetramethyleneethane. Circles denote the ground singlet state, triangles the lowest triplet state, inverted triangles the first excited triplet state and crosses the first excited singlet state.

The spin densities computed in the triplet state show that the terminal sites have a positive spin density and the central sites have a negative spin density. The positive and negative spin densities in the planar geometry are respectively 0.563 and -0.126 and are not sensitive to the twist angle.

The fine structure constants were also calculated using the dipolar spin-spin interactions as perturbations on the exact PPP triplet state for various twist angles. The procedure has been shown to yield results in reasonable agreement with experiment for the triplet states of naphthalene $\left(D=0.114 \mathrm{~cm}^{-1}, E=-0.006 \mathrm{~cm}^{-1}\right.$ and $D=0.167 \mathrm{~cm}^{-1}, E=0.036 \mathrm{~cm}^{-1}$, respectively) (Ramasesha and Soos 1984b; Ramasesha et al 1991). For the planar geometry, $D$ and $E$ were computed to be $0.226 \mathrm{~cm}^{-1}$ and $0.007 \mathrm{~cm}^{-1}$, respectively. In figure 4 is presented the variation of the $D$ and $E$ parameters as a function of the twist angle. For a twist angle of $90^{\circ}$ wherein the molecular symmetry is $D_{2 d}$ the $E$ parameter vanishes as required by symmetry and the $D$ parameter has its lowest value of $0 \cdot 151 \mathrm{~cm}^{-1}$. These values are substantially larger than the recent experimental values (Dowd et al 1986). It is, however, worth noting that the experimental $D$ value was revised upwards by a factor of two by the same group. The computed $D$ value seems more consistent, especially when compared with the values obtained for naphthalene and biphenyl. Spin-spin interactions are expected to be greater in the smaller TME diradical. Thus, the larger $D$ value of TME appears reasonable.

We also computed the $D$ and $E$ values for five other low-lying triplet states of 


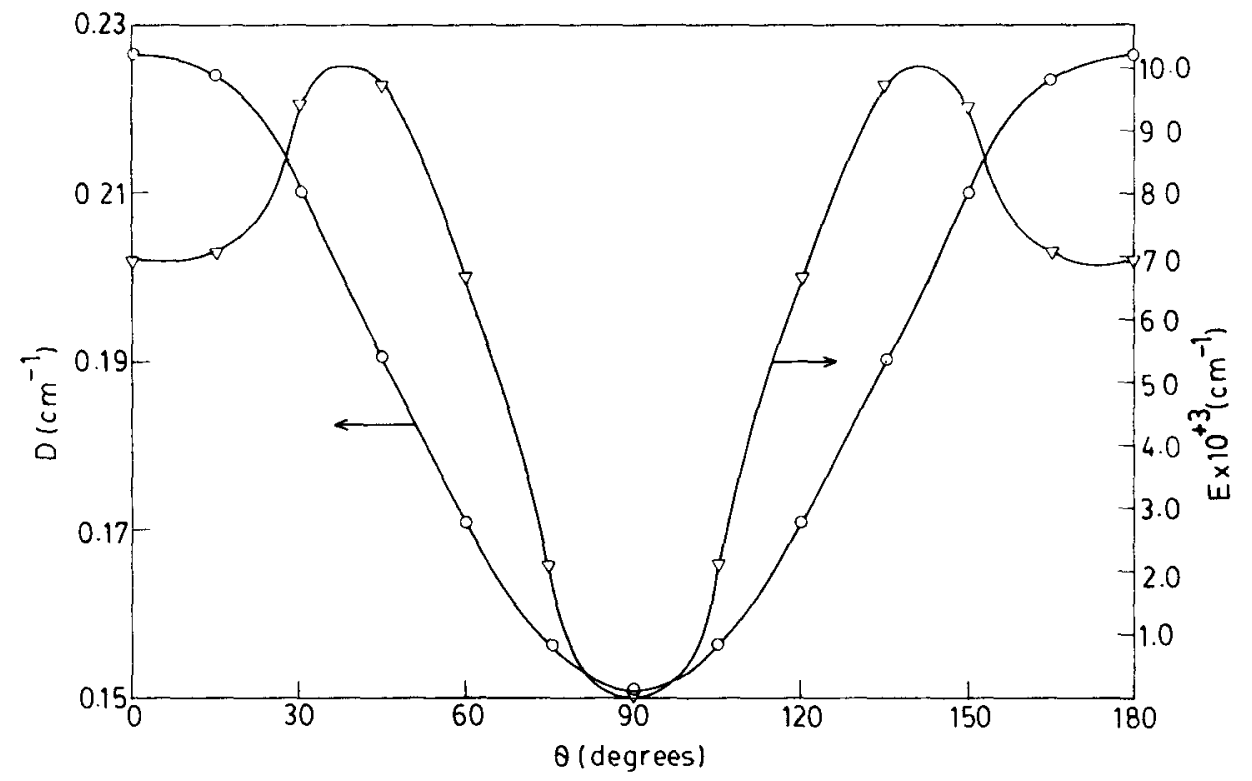

Figure 4. Variation of the fine structure parameters $D$ and $E$ on the twist angle $\theta$ in tetramethyleneethane. Circles represent the fine structure constant $D$ and the inverted triangles the fine structure constant $E$.

TME to consider the possibility of the experimental data corresponding to one of these states. However, we found that the $D$ and $E$ values in the excited triplet states were consistently larger than those computed for the lowest triplet state. Thus it is unlikely that TME was experimentally investigated in a higher energy triplet state.

There are two cyclic conjugated systems with heteroatoms that are closely related to tetramethyleneethane, namely 3,4-dimethylenepyrrole and 3,4-dimethylenefuran. The PPP parameters for both nitrogen and oxygen whose unhybridized lone pairs participate in conjugation are known from the literature (Flurry 1968; Sanchez-Marin and Malrieu 1985). We have used the geometry of their parent compounds and have carried out an exact PPP calculation of these systems (figure 1, II and III). In both the cases, we find that the ground state is a singlet, in agreement with previous $a b$ initio calculations (Du et al 1986) and experiments (Stone et al 1986; Zilm et al 1987). The singlet-triplet gap in these systems is much larger than in tetramethyleneethane. The pyrrole derivative has a gap of $0.694 \mathrm{eV}$ while the furan derivative has a gap of $0.264 \mathrm{eV}$ (table 1). We also find that the gap is larger in the case of pyrrole derivatives in which the heteroatoms participate more in the $\pi$ conjugation. However, in both cases the singlet-singlet gap is similar and so is the gap between the singlet ground state and the second lowest triplet state. In both the singlet and the triplet states of these molecules, bond order calculations indicate that the geometry we have chosen is very close to self-consistency.

Having considered all possibilities by which Ovchinnikov's theorem may be violated within the $\pi$ model, we consider the role of $\sigma-\pi$ polarization effects using the UHF MNDO method. The single determinant UHF procedure for the singlet state yields a wavefunction with substantial spin contamination $\left(\left\langle S^{2}\right\rangle \approx 1\right)$, being a mixture of singlet and triplet components. The computed energy corresponds to that of a 
pseudo-singlet state which may be expected to lie midway between the true-singlet and the triplet. A lower energy for the pseudo-singlet state therefore implies a singlet ground state. This intuitively simple procedure has been found to yield reliable predictions of the ground state of numerous diradicals (Lalitha 1992). Since both the pseudo-singlet and the triplet are treated at the same theoretical level, errors tend to cancel. Further, the key features which determine singlet-triplet energy differences, viz., orbital energy differences, direct overlap effects and dynamic spin polarization, are all adequately described by the computational procedure. A study of TME using the UHF method within the INDO model using assumed geometries has been reported earlier (Hashimoto and Fukutome 1981).

The UHF/MNDO calculations lead to a pseudo-singlet energy $0.22 \mathrm{eV}$ lower than the triplet for planar TME $\left(\Delta H_{f}=2.62\right.$ and $2.84 \mathrm{eV}$, respectively). The ground state is therefore clearly indicated to be a singlet, with a singlet-triplet separation of $\approx 0.44 \mathrm{eV}$. Even for the orthogonal form, the pseudo-singlet energy is lower (energy difference of $0.04 \mathrm{eV}$ ). Thus, the inclusion of $\sigma-\pi$ polarization consistently enhances the stability of the singlet state (relative to that of the triplet) above that predicted by the pure $\pi$-electron models. This is in accord with qualitative expectations. The sites with most spin density in TME are the terminal carbon $p_{\pi}$ orbitals. Dynamic spin polarization of the unpaired electrons occurs via the intervening $3 \mathrm{C}-\mathrm{C} \sigma$ bonds. Generally, the spin-paired arrangement is preferentially stabilized by DSP effects across an odd number of $\sigma$ bonds. Typical examples are orthogonally twisted ethylene (Brooks and Schaefer 1979) and tetramethylene (Borden and Davidson 1980). The same conclusion is arrived at if the spin densities at the central carbon atoms are considered. These are the sites of negative spin density of each of the allyl units. The DSP effect across the central $\mathrm{C}-\mathrm{C}$ bond would ensure that these sites have mutually opposite spin densities. Therefore, the allyl units are predicted to prefer a spin-paired structure, leading to a ground state singlet.

The spin densities obtained by the UHF MNDO calculations are in agreement with the experimental as well as the PPP results. In particular, the magnetic equivalence of the two different kinds of protons of TME found in the EPR spectrum is borne out by the equal negative spin densities computed at the corresponding hydrogen centres. Further, similar data for the TME radical cation and anion are also consistent with the experimentally observed proton hyperfine coupling constants (Bauld and Stevenson 1969; Gerson et al 1989). The pleasing agreement between the computational and experimental spectral results confirms that the EPR study of TME has indeed been carried out on the lowest energy triplet. However, energetically, this triplet state lies above the lowest singlet state even at the UHF MNDO level.

All the calculations lead us to conclude that the ground state of TME is a singlet and is stable with respect to a variety of perturbations. How does one reconcile these results with the conclusion based on careful experimental studies? We suggest a possible explanation to resolve the conflict. The photochemical means of generating TME (or its analogues) by high energy laser radiation could produce the diradical in its triplet excited state. At $15 \mathrm{~K}$, the triplet may be sufficiently long-lived. Increase in temperature would then deplete its population due to thermal degradation. Consequently, the EPR spectral intensity would decrease in an apparently Curie-Weiss manner. Although these are conjectures, they are amenable to an experimental test. If they are correct, the temperature dependence of the EPR spectral intensity will not be reversible with respect to recycling the temperature. A critical 
examination of the reversibility of magnetization change with temperature as well as a study over a wider temperature range is required to resolve the conflict between theory and experiment.

\section{Acknowledgement}

IDLA and SL thank the Council of Scientific and Industrial Research, New Delhi, for fellowships. AC thanks the University Grants Commission, New Delhi, for a fellowship.

\section{References}

Albert I D L, Ramasesha S and Das P K 1991 Phys. Rev. B43 7013

Bauld N L and Stevenson G R 1969 J. Am. Chem. Soc. 9113

Borden W T 1982 Diradicals (ed.) W T Borden (New York: Wiley) pp. 1-72

Borden W T and Davidson E R $1977 \mathrm{~J}$. Am. Chem. Soc. 994487

Borden W T and Davidson E R 1980 J. Am. Chem. Soc. 1025409

Brooks B R and Schaefer H F 1979 J. Am. Chem. Soc. 101307

Choi Y, Jordan K D, Paik Y H, Chang W and Dowd P 1988 J. Am. Chem. Soc. 1107575

Coulson C A 1961 Proc. R. Soc. A169 407

Dewar M J S and Thiel W 1977 J. Am. Chem. Soc. 99 4899, 4907

Dowd P, Chang W and Paik Y H $1986 \mathrm{~J}$. Am. Chem. Soc. 1087416

Dowd P, Chang W and Paik Y H 1987 J. Am. Chem. Soc. 1095284

Du P and Borden W T 1987 J. Am. Chem. Soc. 109930

Du P, Hrovat D A and Borden W T 1986 J. Am. Chem. Soc. 1088086

Ducasse L R, Miller T E and Soos Z G 1982 J. Chem. Phys. 764094

Flurry R L (Jr.) 1968 Molecular orbital theories of bonding in organic molecules (New York: Marcel Dekker)

Gerson F, Armin De Meijere and Xue-Zhi Qin 1989 J. Am. Chem. Soc. 1111135

Hashimoto K and Fukutome H 1981 Bull. Chem. Soc. Jpn. 543651

Hubbard J 1963 Proc. R. Soc. A276 276

Kuwajima S and Soos Z G 1987 J. Am. Chem. Soc. 109107

Lahti P M, Rossi A R and Berson J A 1985 J. Am. Chem. Soc. 1072273

Lalitha $\mathrm{S} 1992 \mathrm{Ph} \mathrm{D}$ thesis, Indian Institute of Science, Bangalore

Lieb E H and Mattis D C 1962 Phys. Rev. 125164

Ohno K 1964 Theoret. Chim. Acta 2219

Ovchinnikov A A 1971 Theor. Chim. Acta 47297

Pariser R and Parr R G 1953 J. Chem. Phys. 21767

Pople J A 1953 Trans. Farad. Soc. 421375

Radhakrishnan T P 1991 Chem. Phys. Lett. 181455

Ramasesha S, Albert I D L and Sinha B 1991 Mol. Phys. 72537

Ramasesha S and Soos Z G 1984a J. Chem. Phys. 803278

Ramasesha S and Soos Z G 1984b Chem. Phys. 9135

Roth W R, Kowlczik U, Maier G, Reisenauer H P, Sustmann R and Muller W 1987 Angew. Chem., Int. Ed. Engl. 261285

Sanchez-Marin J and Malrieu J P 1985 J. Am. Chem. Soc. 89978

Sinha B, Albert I D L and Ramasesha S 1990 Phys. Rev. B42 9088

Soos Z G and Ramasesha S 1983 Phys. Rev. Lett. 512374

Stone K J, Greenberg M M, Goodman J L, Peters K S and Berson J A 1986 J. Am. Chem. Soc. 1088088

Zilm K W, Merrill R A, Greenberg M M and Berson J A 1987 J. Am. Chem. Soc. 1091567 